International Journal of Wireless \& Mobile Networks (IJWMN) Vol. 10, No. 4, August 2018

\title{
Particle Filter Approach to Utilization of Wireless Signal STRENGTH FOR MObILE Robot LOCALIZATION IN INDOOR ENVIRONMENTS
}

\author{
Samuel L. Shue ${ }^{1}$, Nelyadi S. Shetty ${ }^{1}$, Aidan F. Browne ${ }^{1}$ and James M. Conrad ${ }^{1}$ \\ ${ }^{1}$ The William States Lee College of Engineering, \\ The University of North Carolina at Charlotte, \\ Charlotte, North Carolina, USA
}

\begin{abstract}
For many autonomous robotic applications, the capability to simultaneously create a map of the environment while localizing its position within it is of critical importance. This is typically achieved by fusing odometry information from the robotic vehicle with information from landmarks detected within the environment. Indoor environments often have existing wireless infrastructure, which can be used as landmarks by estimating the distance between the robot and the access point. The most practical way to attain this is by measuring the decay of signal strength. However, radio signal strength does not predictably attenuate indoors as it does in open environments due to signal interference, absorption, and reflection from objects within the environment, inflicting unexpected amplification or decay at the receiver known as multipath interference. This causes erroneous distance estimations due to the unexpected changes in signal strength attenuation. In this research, models of radio propagation as it relates to the received signal strength indicator (RSSI) are explored along with localization techniques which utilize these models. For development and testing of RSSI-based localization techniques, a simulation method has been described which utilizes a Markov chain to provide realistic multipath interference on simulated RSSI data. Using this simulation technique, methods for simultaneous localization and mapping (SLAM) are explored. Due to the difficulty associated with modeling RSSI attenuation and distance estimation, a particle filter based SLAM approach is proposed and demonstrated.
\end{abstract}

\section{KEYWORDS}

RSSI, ZigBee, Localization, Particle Filter, SLAM \& RO-SLAM

\section{INTRODUCTION}

In recent years, mobile robotics has grown into an enormous research field for both industrial and domestic applications. These applications include autonomous vacuum cleaners, pool cleaners, lawn mowers, and self-driving vehicles, just to name a few. In all of these applications, knowledge of the robot's position within its operating environment is of critical importance. To accurately fulfill the requirements of any mobile robotics task, the robot must be aware of its position relative to the objects within the environment to properly interact with them. The robot must also be able to build a map to assist in navigating from point to point within that area. This presents a causality dilemma, in which the robot must know its own position to build a map, but also know the map to track its position. Algorithms that attempt to solve this problem are known as Simultaneous Localization and Mapping (SLAM) algorithms.

Robots use a variety of sensors to estimate their position, such as odometry measurements from the mobile base as well as range finding sensors or visual information from cameras to help 
identify recognizable landmarks within the environment. In this research, wireless signal strength is used as sensory information to estimate the distance between transceivers. If the robot is equipped with a wireless transmitter, as the signal attenuates while traveling to the receiver, distance to that receiver can be estimated based on the loss of signal strength [1]. This loss of power between transceivers is represented by the Received Signal Strength Indicator (RSSI). Most wireless transceivers represent RSSI in $-\mathrm{dBm}$ (decibel milliwatts) which represents the amount of attenuation or lost power during transmission. If the operating environment of a wireless mobile robot is equipped with transceivers scattered throughout, the estimated distance to each transceiver from the robot can be used to refine the position estimation of the robot $[2,3]$.

There are several mathematical models which can be used for estimating a distance from RSSI. These models largely do not account for the multipath fading effect. Multipath fading occurs when a signal takes multiple paths from the transmitter to the receiver. Depending on the phase of signals when they arrive together, they can either cause either large attenuation or large amplification of the signal. When the signal is suddenly amplified, the model will not hold and will generate highly erroneous readings. Some models account for multipath but are intended for long-range transmissions, such as transmission from a cell tower [4, 5, 6]. Multipath fading effects in short-range indoor environments are much more difficult to predict due to the many factors that affect its behavior. For mobile robot operating environments, such as a warehouse or a private home, short-range multipath is the most prevalent problem in RSSI distance estimation.

Many solutions exist to mitigate the effects of multipath attenuation. However, most of these rely on some modification to the wireless modulation hardware. Common strategies involve analyzing the signal before the demodulation phase, attempting to identify multipath components and removing them, leaving the primary path intact. Ultra-Wideband techniques utilize various frequencies for transmitting the same message and observing how each frequency is affected by the transmission, allowing the detection and compensation for multipath interference. Other techniques will utilize antenna arrays or directional antennas to avoid multipath interference. While these methods have proven effective, they all require some specialty modification to the transmission and reception hardware or utilize non-standard communication protocols. This limits the use of these techniques in existing networks and an increase in implementation costs due to hardware obscurity. For this reason, it would be desirable to find a solution that utilizes the common hardware. Most wireless devices provide the user with RSSI values for each transmission. If multipath interference could be accounted for in RSSI-based ranging techniques, localization could be accomplished in a more inexpensive and accessible manner [2].

\subsection{Objective}

In this work, wireless transceivers will be utilized as landmarks for SLAM algorithms with a focus on indoor environments. SLAM algorithms use the robot's odometry information along with detected landmarks within the environment to estimate the position and orientation of the robot as well as building a map using range data typically from a laser rangefinder or camera. To effectively correct odometry errors, SLAM algorithms typically need to collect and maintain many landmarks. This tends to cause most SLAM algorithms to scale poorly over time by consuming exponentially more memory as new landmarks are appended. Another issue encountered through the use of landmarks is the data association problem, where a newly observed landmark may be improperly identified as a previously recorded, different landmark.

Utilizing wireless transceivers as landmarks largely solve these problems, since the number of transceivers is finite, limiting the number of landmarks to the number of devices within the network. Generally, there are not enough network devices where the memory consumption becomes a problem for most modern computers and embedded devices. The landmark association problem is entirely solved as each landmark has a network address associated with it. However, 
the multipath problem encountered when using wireless beacons renders many common SLAM algorithms inappropriate, such as algorithms based on the Extended Kalman Filter (EKF), which requires measurement noise to be well modeled by a zero-mean Gaussian distribution.

\subsection{Contribution}

This paper proposes a particle filtering SLAM configuration to properly handle the non-Gaussian distributed data provided by RSSI range estimation. This method is validated through a simulation which utilizes a unique RSSI simulation technique to provide realistic multipath interference and noise distribution. The results are then contrasted with the standard EKF rangeonly SLAM methodology.

\section{BACKGROUND}

\subsection{RSSI Ranging}

Distance estimation through signal strength decay is one of the most practical methods as most wireless hardware interfaces provide RSSI as an accessible parameter. Multiple models exist to describe the signal attenuation over distance, however, the most commonly used model is the logdistance path loss model. The log-distance path loss model is a general propagation model. It can be used in both indoor and outdoor environments. The log-distance path loss model provides a logarithmic attenuation model which has several parameters that can be tuned to make it fit nearly any environment $[7,8]$. The RSSI (in $\mathrm{dBm}$ ) for this model is expressed as:

$$
R S S I=10 n \log _{10} d+A
$$

Where $\mathrm{n}$ is the path-loss exponent, $d$ is the transmission distance in meters, and $A$ is the reference value, which is the RSSI at 1 meter away from the transmitter. This equation can be rearranged to be expressed as a distance for a given RSSI value:

$$
d=10^{\frac{R S S I-A}{10 n}}
$$

The path loss exponent, $n$, can be calculated for each environment by recording RSSI values at known distances and reverse solving for $n$. Typical values for $n$ can be observed in Table I [8, 9, $10]$.

Table 2.1: Sample path-loss exponent values for various environments.

\begin{tabular}{cc}
\hline Environment & $\begin{array}{c}\text { Path-Loss Exponent } \\
\text { (n) }\end{array}$ \\
\hline Free Space & 2.0 \\
Urban Area Cellular Radio & $2.7 \sim 3.5$ \\
In-Building LOS & $1.6 \sim 1.8$ \\
Shadowed Urban Area & $3 \sim 5$ \\
Cellular Radio & \\
\hline
\end{tabular}

The log-distance path loss model is the most versatile, as it can be configured for each environment and uses a reference value rather than requiring the transmission power and gain for each transmitter and receiver. 


\subsection{EKF SLAM}

The Extended Kalman Filter (EKF) SLAM is one of the most common and reliable implementations of SLAM. It can be adapted for multiple types of landmarks, can be quickly computed, and has a relatively straight-forward implementation. As the name implies, it uses the EKF at its core for handling the uncertainties associated with SLAM. EKF is a recursive Bayesian filter which models each input and prediction with zero mean Gaussian noise [10].

\subsection{Particle Filter}

Particles filters are a category of Monte Carlo algorithms that are used to estimate states in partially observable Markov chains [11]. Monte Carlo algorithms represent the distribution of a random variable through a large number of samples. This non-parametric method of representing a distribution is appropriate for situations such as RSSI-distance estimation in an indoor environment, where the distribution of the noise is nearly impossible to model.

The particle filter represents the state of the system through a dataset, $X_{t}$, composed of $M$ random samples known as particles. Each particle, $x_{t}$, represents the hypothesis of that particular state variable at time $t$. Like the Kalman filter, the particle filter can be represented with two basic steps: prediction and measurement/re-sampling. The prediction phase uses a control input, $u_{t}$, to updates each particle's value, $x_{t}^{[m]}$, and posterior distribution, $p\left(z_{t} \mid x_{t}\right)$, based on system dynamics. The measurement phase uses sensory information, $z_{t}$, to validate the prediction of each particle. The error between the measured sensory information and the predicted state is computed for each particle, and a weight, $w_{t}$, is generated based on that error. The particles in the filter are then randomly sampled according to the distribution formed by the weight assigned to each particle (particles with predicted states that closely matched the measured state are assigned a higher weight and are more likely to be selected). The particles are resampled $M$ times, once for each particle in the filter. Over many iterations, the particles converge towards the true state of the system $[11,12]$. The algorithm used for the Particle Filter $\left(X_{t-1}, z_{t}\right)$ is as follows:

$$
\begin{aligned}
& \text { 1: } \bar{X}_{t}=X_{t}=0 \\
& \text { 2: for } m=1 \text { to } M \text { do } \\
& \text { 3: } \quad \text { sample } x_{t}^{[m]} p\left(x_{t} \mid u_{t}, x_{t-1}^{[m]}\right) \\
& \text { 4: } \quad w_{t}^{[m]}=p\left(z_{t} \mid x_{t}^{[m]}\right) \\
& \text { 5: } \quad \bar{X}_{t}=\bar{X}_{t}+<X_{t}^{[m]}, w_{t}^{[m]} \\
& \text { 6: for } m=1 \text { to } M \text { do } \\
& \text { 7: } \quad \text { draw i with probability } \approx w_{t}^{[i]} \\
& \text { 8: } \\
& \text { 9: return } X_{t}
\end{aligned}
$$

Particle filters have been successfully able to solve applications in the robotics domain for more than one reason. They can be applied to any model that is probabilistic and can be represented as a Markov chain. Secondly, particle filters do not require a fixed computational time rather their accuracy increases with increase in computational resources as mentioned in [13] and [14]. 

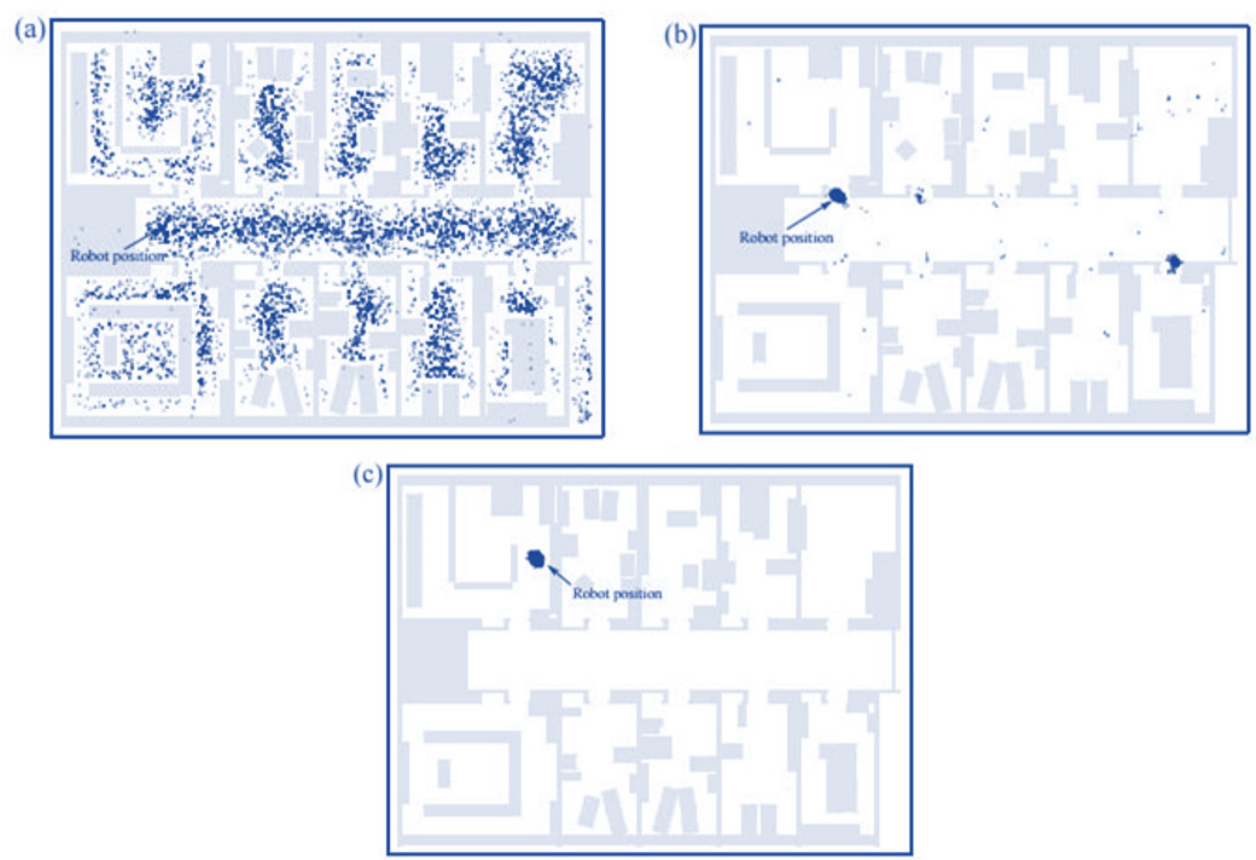

Figure 1: Convergence of particle filter for mobile robot localization [11].

\section{Methodology}

\subsection{Implementation}

This research proposes a pure particle filter RO SLAM implementation for RSSI ranging application. This implementation describes each aspect of the SLAM algorithm with individual particle filters. Initially, a particle filter is defined for the location and orientation of the mobile robot. As with most SLAM algorithms, the robot is defined initially and the map is created with the origin centered on the robot's starting position. These particles begin with a high certainty centered on this location. As the robot receives control inputs, $u_{t}$, from the odometry the particles and their posteriors are computed. However, the resampling phase cannot be computed until landmarks are identified within the environment.

When the robot receives a new RSSI reading from an uninitialized landmark, a new particle filter is generated for that landmark. Unlike the robot, the landmarks have a highly uncertain position. Therefore, the particles are initialized randomly from a uniform distribution across the entire region of interest. The landmarks have no control input, but slowly converge as measurements are taken by the mobile robot. The expected range value is computed for each particle using the expected value from the mobile robot particle filter and the hypothesized location of the landmark for that particle.

Once a landmark particle filter has converged to an acceptable level, it can be used to resample the mobile robot particle filter. The robot's location particles are resampled in a similar manner to the landmarks, using the expected value of the landmark particles to generate the expected values for each particle. This process is detailed in the following implementation algorithm: 


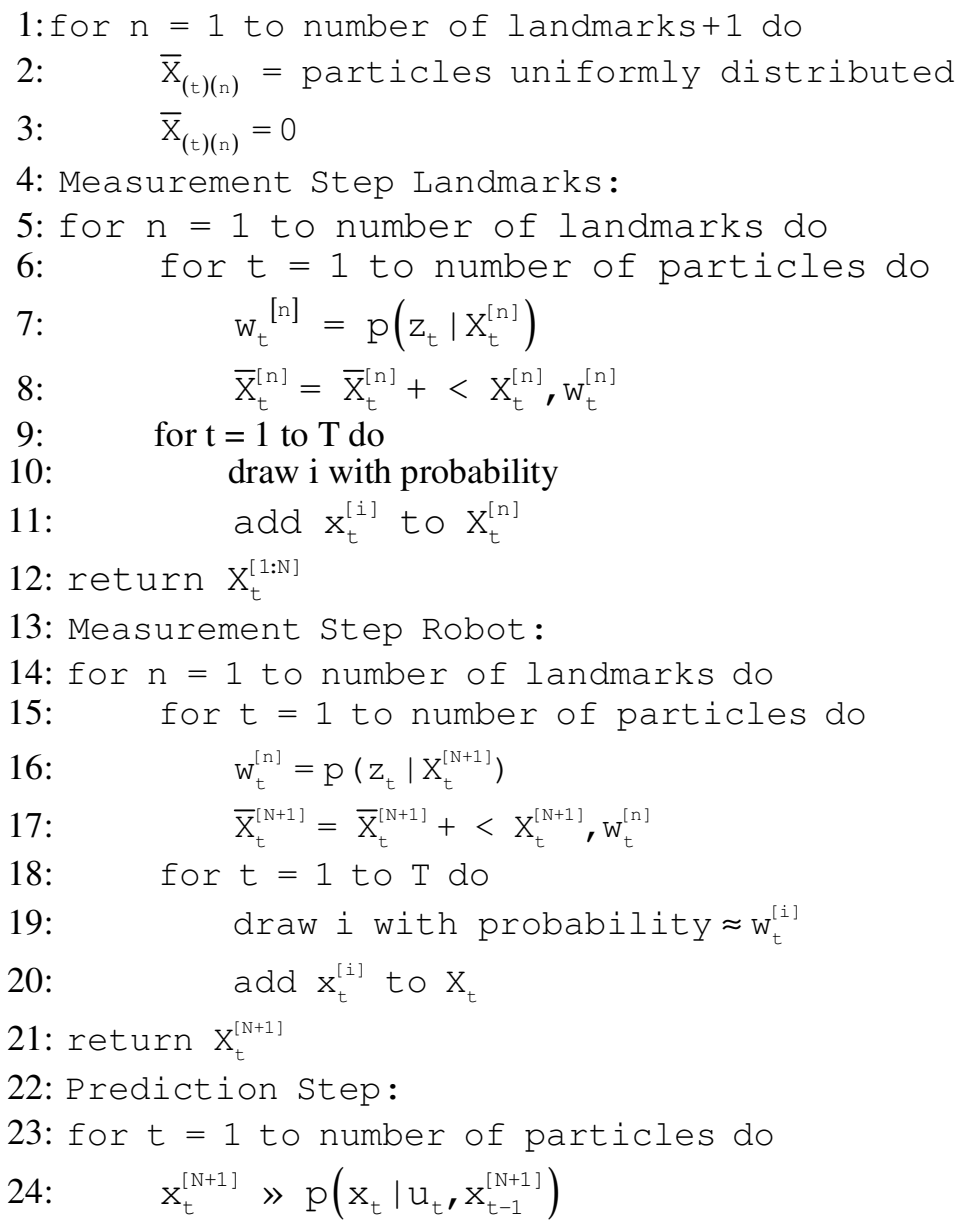

As with all RO SLAM algorithms, a landmark must go through an initialization phase prior to being used for measurement. This is accomplished in this implementation by waiting until the particles for a landmark have converged to an acceptable level. However, when using RSSI ranging, it is possible for a landmark to converge while undergoing multipath interference, creating a highly erroneous initialization. When this occurs in EKF based algorithms, it can be difficult to recover the landmark, as the Gaussian assumption prevents the estimation from rapidly updating when leaving the area undergoing the multipath interference. The particle filter's adaptive nature makes this scenario less impactful on the overall state of the filter.

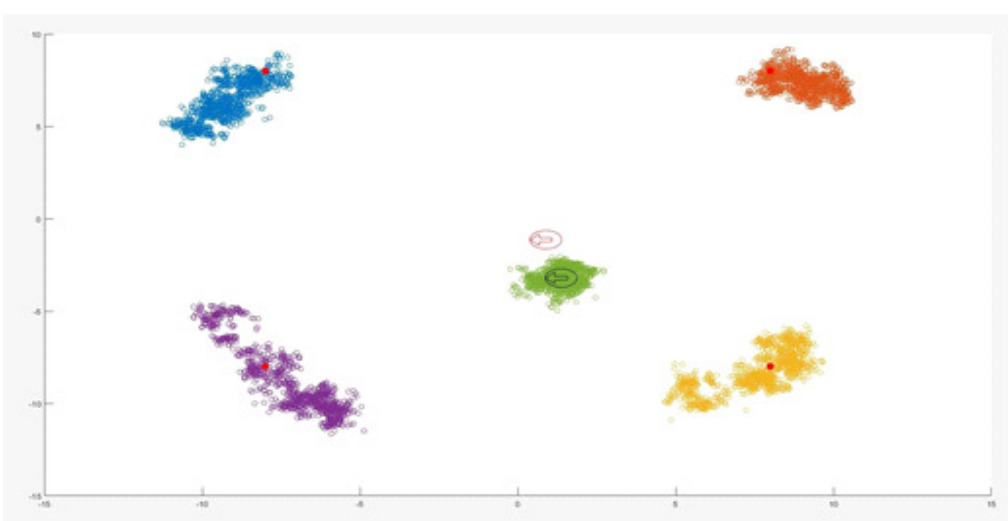

Figure 2: Implementation of Particle Filter used to perform SLAM. 
The red dots and red circle are the actual landmark and robot positions. The blue, purple, yellow, orange particles represent each landmark. The green set of particles represent the robot particle filter and the black circle represents the expected value of the robot pose.

\section{RSSi SimUlation METHOD}

An RSSI simulation method was used to create the environment used to evaluate the method described in the previous section. This simulation method utilizes a Markov chain trained on collected RSSI data to produce an RSSI-distance dataset. The Markov chain generates a lookup table that is used to access an RSSI value when the robot is at a specific position relative to the wireless node. This simulation method captures the complex effects of multipath interference without the need the model the environment, which is usually a tedious and computationally expensive process as multipath is determined by not only the geometry of the environment but also the reflectiveness of the materials which compose it. This simulation is described in detail in [15].

For the examples shown in this work, the Markov chain was trained on data collected within the Energy Production and Infrastructure Center (EPIC) building on the campus of the University of North Carolina at Charlotte. Data were collected in various environment types, including large classrooms, hallways, and laboratories. An outdoor dataset was also collected for a set with minimal multipath interference. This data was collected using XBee 802.15.4 radios and Digi 2.4 $\mathrm{GHz}$ Omnidirectional Dipole antennas with a $2.1 \mathrm{dBi}$ Gain. The XBee radios were configured with boost mode disabled and power level set to 2 (1 dBm Gain). Each XBee module was interfaced with an Arduino Uno; one module being set to transmit, and the other configured to return the RSSI value of the received packet. Each node was placed on a 1 meter PVC pole and displaced from the transmitter at 0.25-meter increments up to 10 meters. At each increment, 5 readings were taken at 90 -degree increments for a full rotation, to account for any asymmetric radiation patterns of the antennae and its effect on the RSSI reading $[15,16]$.

\section{RESULTS}

Using the RSSI simulation method described in 3.2, simulation environments were created to validate the proposed particle filter method. Different test cases were simulated for various RSSI datasets used. The test cases for each environment are further divided into landmarks initialized at true locations and falsely/erroneously initialized landmarks. Both cases use the same set of RSSI signals generated by our simulator. The first graph in each case represents the distribution of the noise with multipath from the radio beacons. This is obtained through the difference between the actual robot and landmark to the distance derived from the signal generated by our simulator. The following graphs represent the accumulated robot position error for the different methods, landmark localization error and the actual path followed by ground truth, dead reckoning, EKF RO SLAM, and the proposed Particle Filter RO SLAM. In each case, the robot is driven with a constant linear velocity of $0.1 \mathrm{~m} / \mathrm{sec}$ in an S-Shaped track and an angular velocity of 30 degrees at the turns. To save computation time, it is assumed the landmarks have already been initialized/converged with some amount of error. To ensure each particle filter used in the proposed method represents the distribution well, 1000 particles are generated for each filter in these simulations. 


\subsection{Large Open Room Environment}

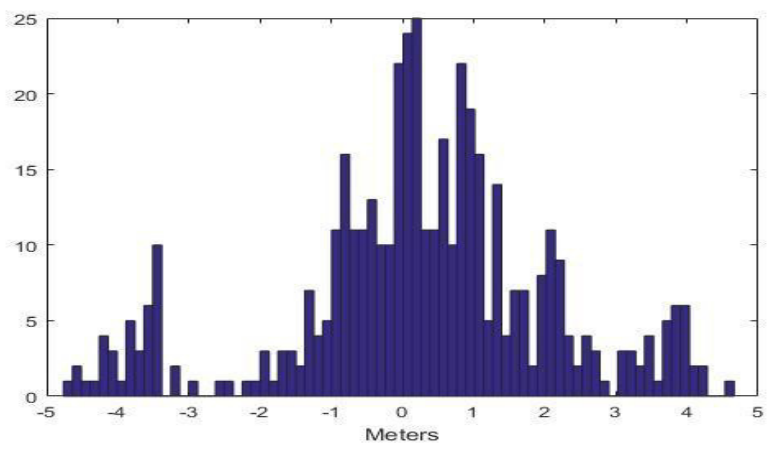

Figure 3: Error in distance derived from RSSI(in meters) in a large open room environment.

Figure 3 displays the histogram of the beacon noise which has a variance of 3.5 meters. This value is assigned as the variance of the measurement noise in RO EKF. Also, a Gaussian zero mean odometry noise with a variance of 0.1 meters linear velocity is introduced, a zero mean Gaussian noise of 10 degrees is used for the angular velocity noise. It uses a particle filter that maintains 1000 particles for each landmark and robot.

\section{True Landmark Initialization}

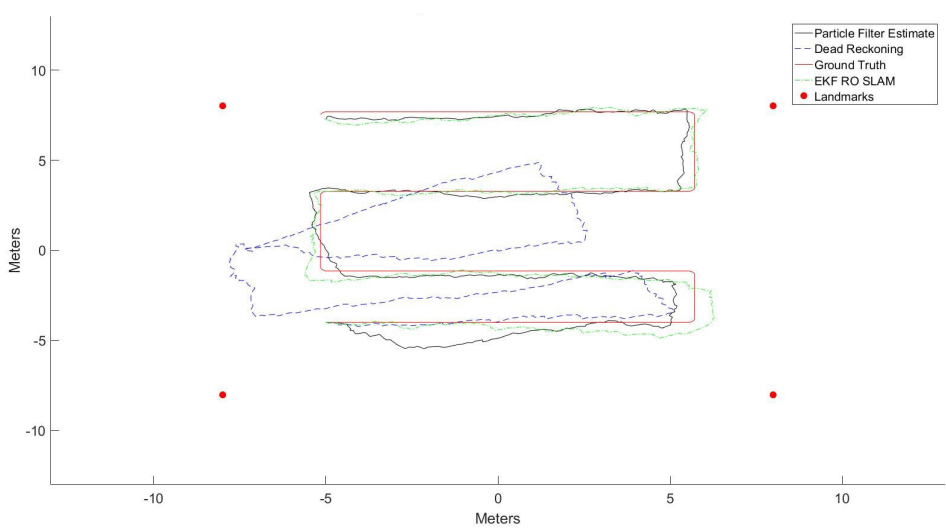

Figure 4: Error in the path followed in a large open room environment.

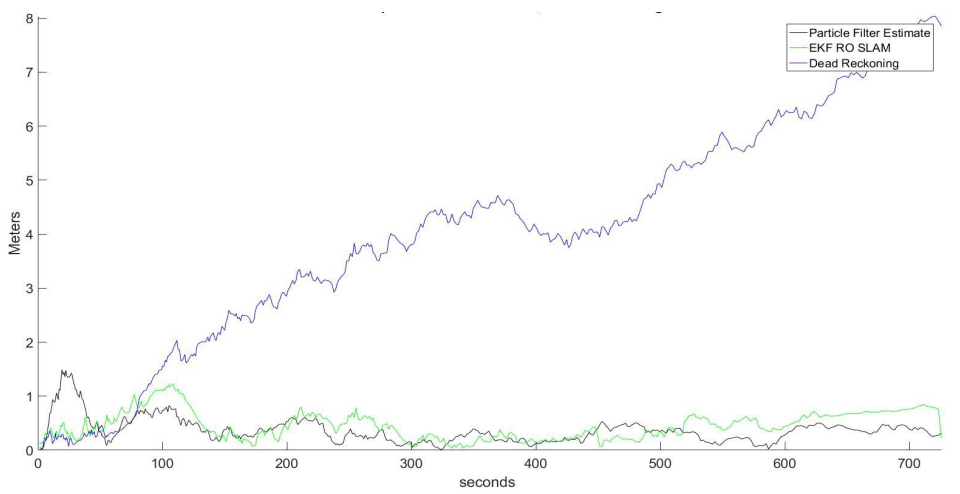

Figure 5: Error in position accumulated over the path in a large open environment over time. 
International Journal of Wireless \& Mobile Networks (IJWMN) Vol. 10, No. 4, August 2018

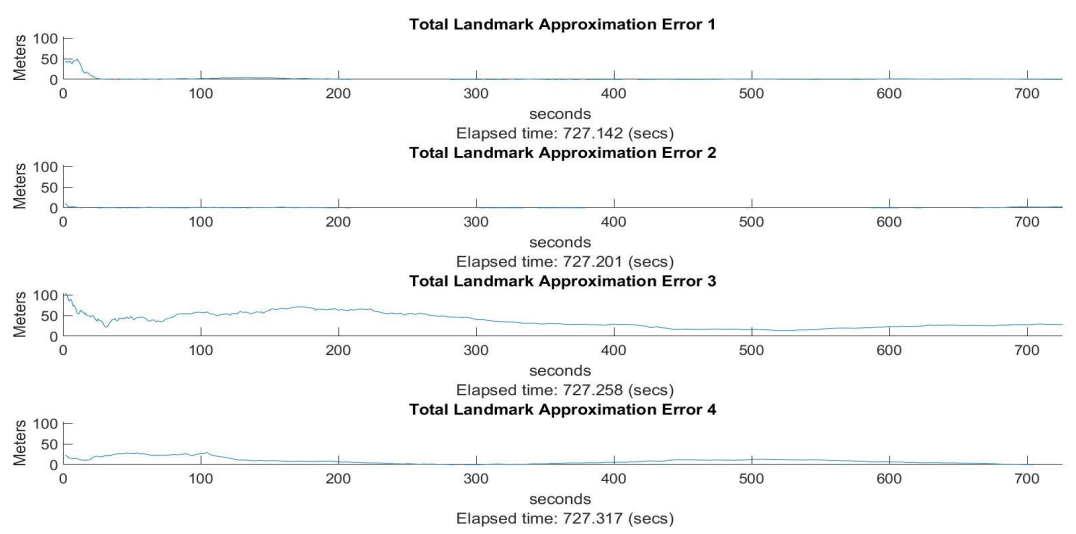

Figure 6: Error in the landmark localization in a large open room environment over time.

In this simulation, the multipath interference is minimal for an indoor environment, which is reflected in Figure 5 where the location error between the particle filter method and RO EKF are similar and low. This is due to the fact the noise in the large open room has a better Gaussian approximation than what was observed in the following experiments. With minimal multipath interference on the signal, it is expected for RO-EKF and the particle filter to exhibit comparable performance.

\subsubsection{False Landmark Initialization}

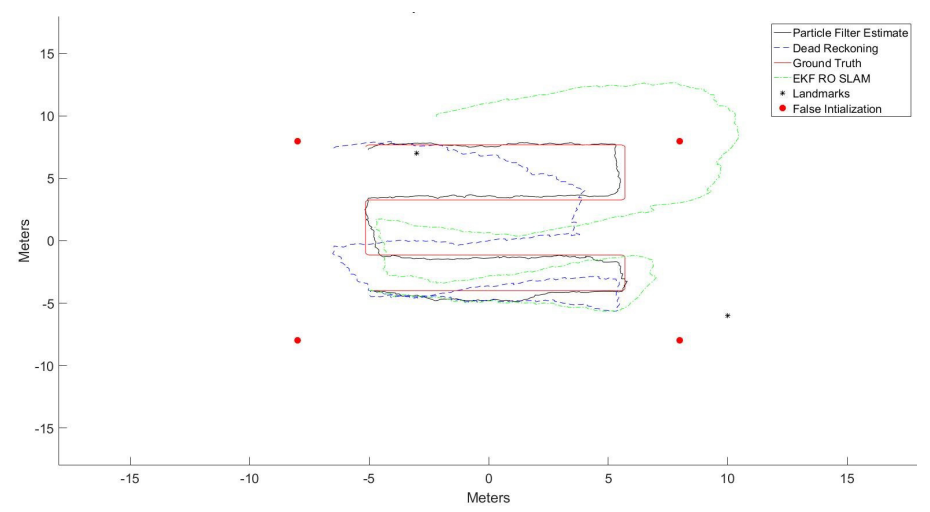

Figure 7: Error in the path followed in a large open room environment with false initialization.

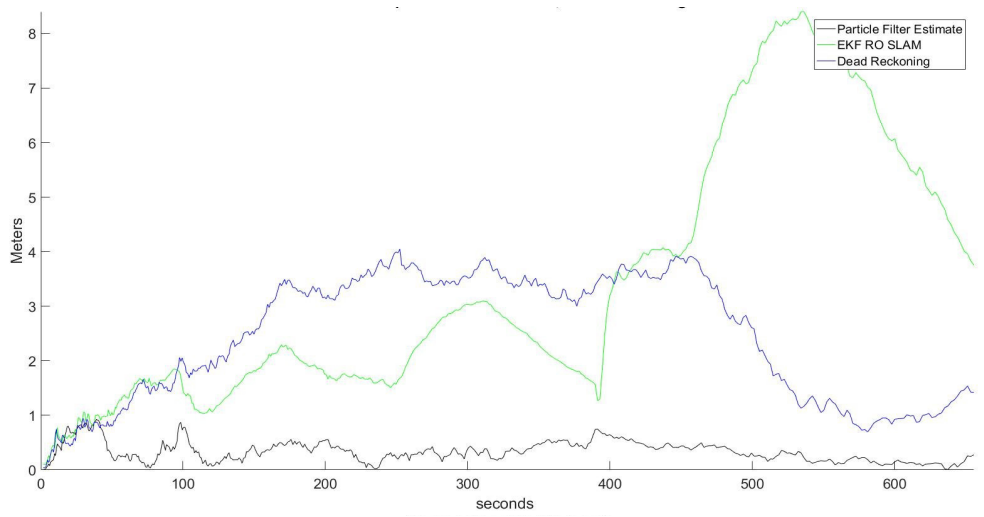

Figure 8: Error in the robot's position in a large open room environment with false initialization over time. 
International Journal of Wireless \& Mobile Networks (IJWMN) Vol. 10, No. 4, August 2018

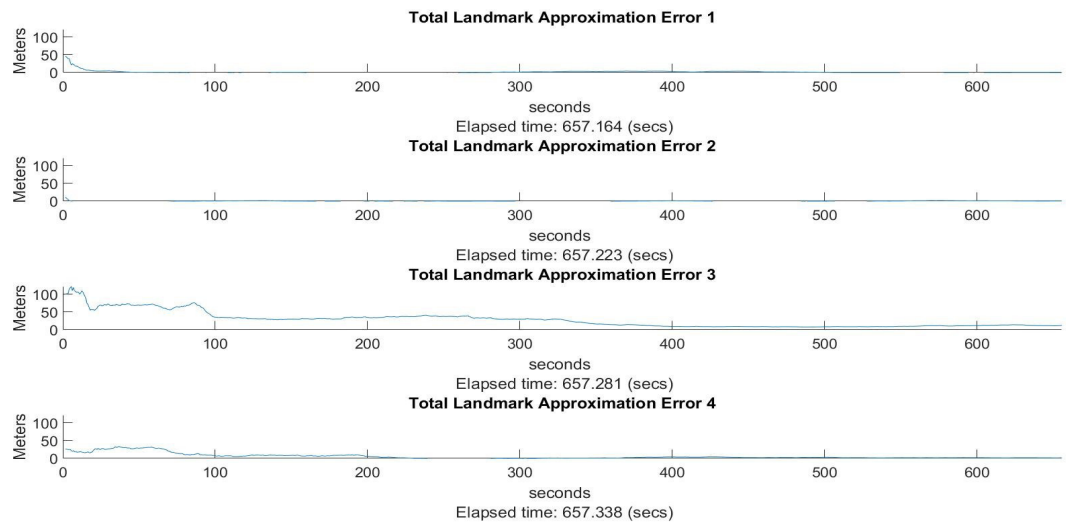

Figure 9: Error in the landmark localization in a large open room environment with false initialization over time.

In this experiment, the landmarks are given incorrect initial conditions when added to each SLAM algorithm, as shown in Figure 7. Despite the RSSI noise being near-Gaussian, the approximation does not serve well when attempting to correct faulty landmark initialization. The faulty landmarks and inappropriate Gaussian noise representation causes the false landmarks to generate faulty corrections to the robot's pose, causing the filter to become more erroneous over time.

\subsection{Hallway Environment}

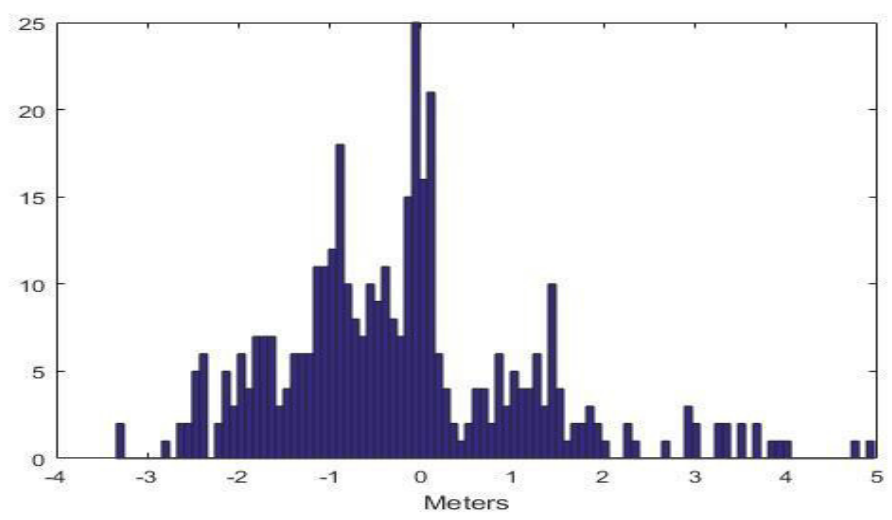

Figure 10: The histogram of the error in RSSI signals (in meters) generated by the simulator by using the dataset representing hallway environments.

In this case, a measurement noise of 3.25 meters variance is applied to RO EKF SLAM measurement noise and repeated the process mentioned in the previous test case. 
International Journal of Wireless \& Mobile Networks (IJWMN) Vol. 10, No. 4, August 2018

\subsubsection{True Landmark Initialization:}

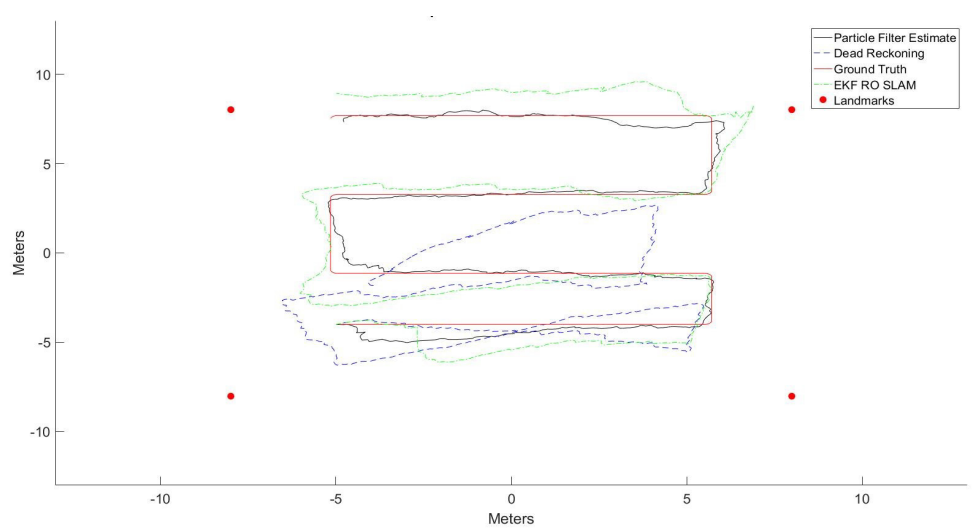

Figure 11: Error in the path followed in Hallway environments.

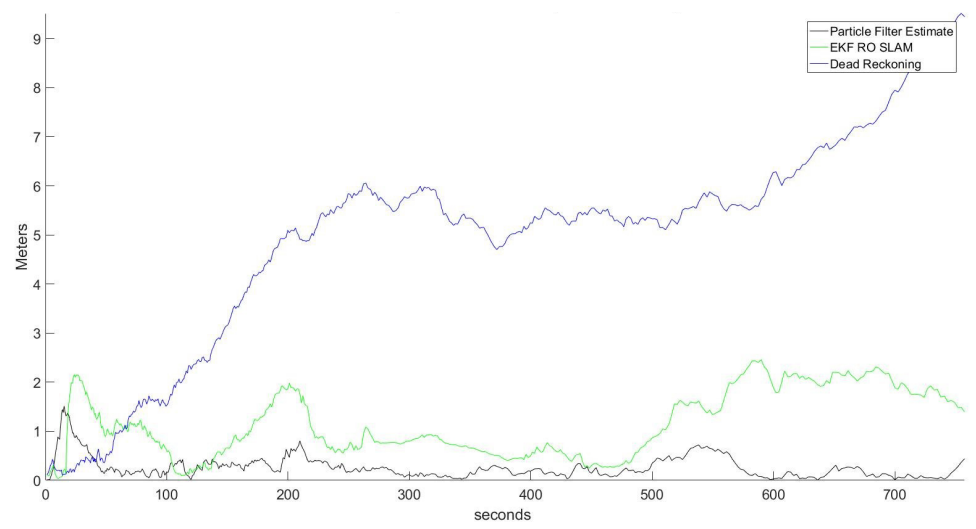

Figure 12: Error in the robot's position accumulated over the entire path in a Hallway environment.
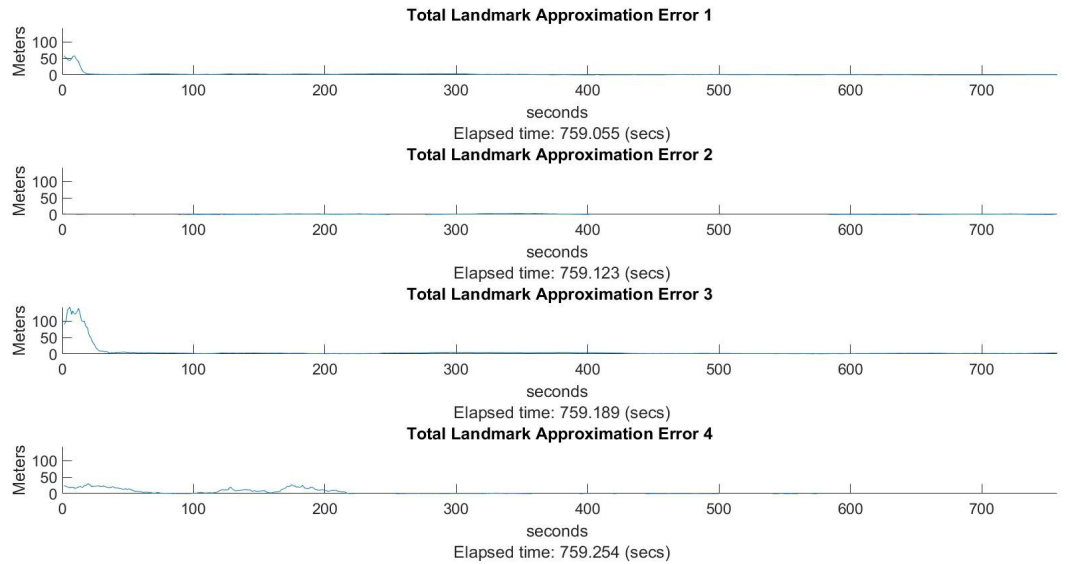

Figure 13: Error in the landmark localization in a Hallway environment. 
The hallway environment has a poorly approximated Gaussian noise distribution, which creates huge problems for the RO-EKF method. Here, the particle filter method is able to adapt to the shifts in noise distribution from multipath interference, keeping the localization error below $\sim 1$ meter, which is to be expected for RSSI applications.

\subsubsection{False Landmark Initialization}

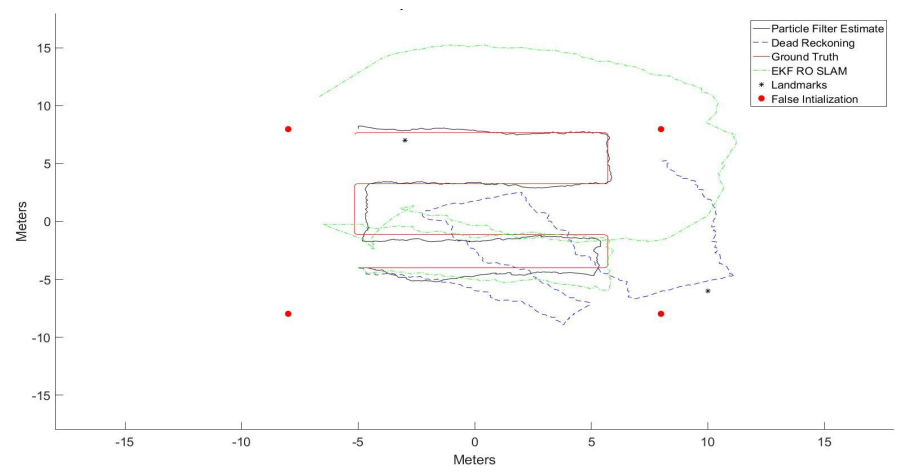

Figure 14: Error in the path followed in a Hallway environment with false initializations.

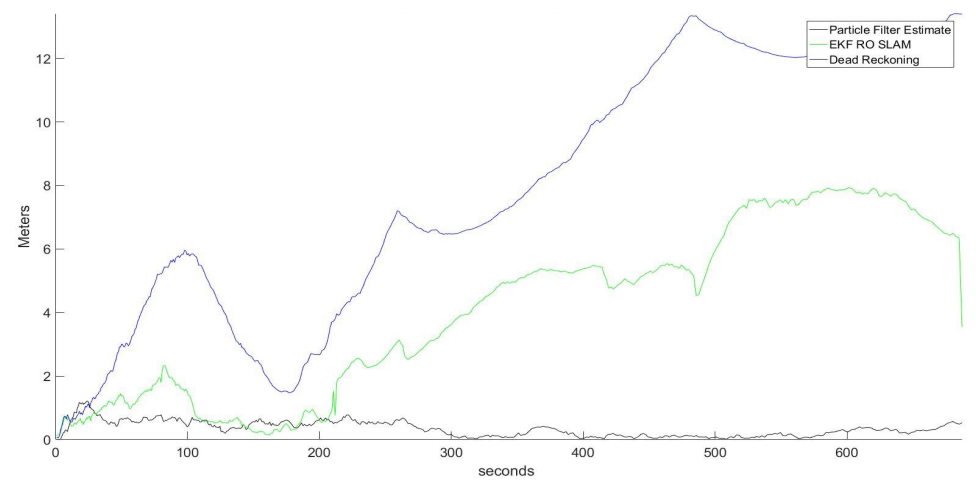

Figure 15: Error in the robot's position in a Hallway environment with false initialization.
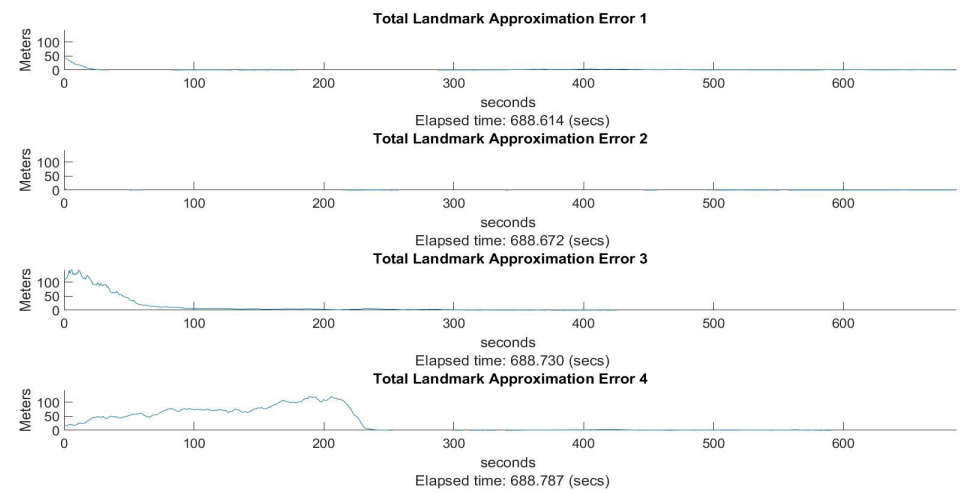

Figure 16: Error in the landmark localization in a Hallway environment with false initialization.

The hallway experiment with faulty landmark initialization proves even more troublesome for RO-EKF, causing erroneous measurements and noise representation to accrue rapidly. The particle filter robot pose location error remains similar to the previous experiment, however, it is also able to correct the beacon locations as well. 


\subsection{Furnished laboratory environment}

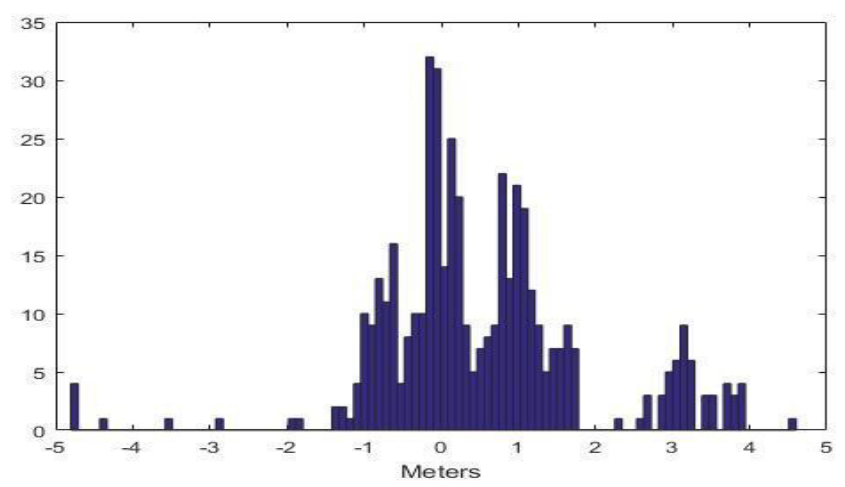

Figure 17: The histogram of the error in RSSI signals (in meters) generated by the simulator by using the dataset representing a lab.

In this case, we have used a measurement noise of 2 meters variance in our RO EKF and have repeated the process mentioned in the previous test case.

\subsubsection{True Landmark Initialization}

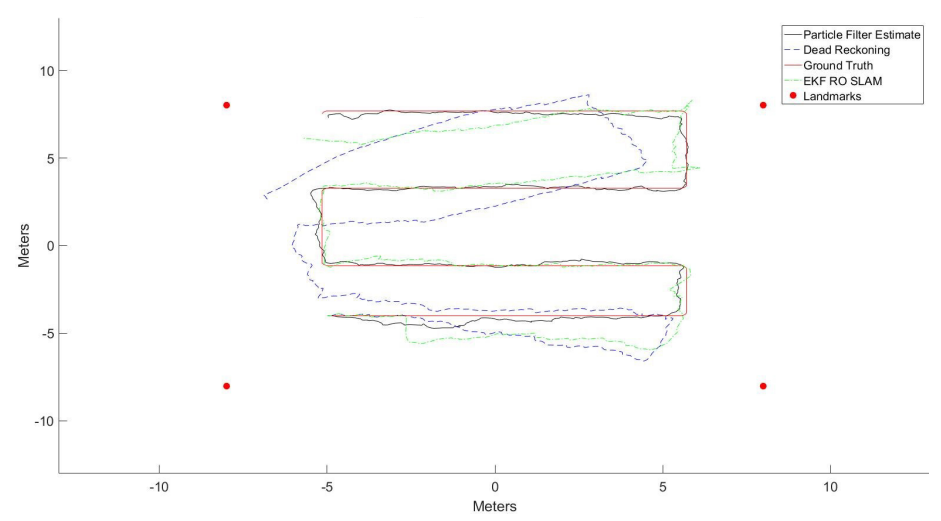

Figure 18: Error in the path followed in a lab environment.

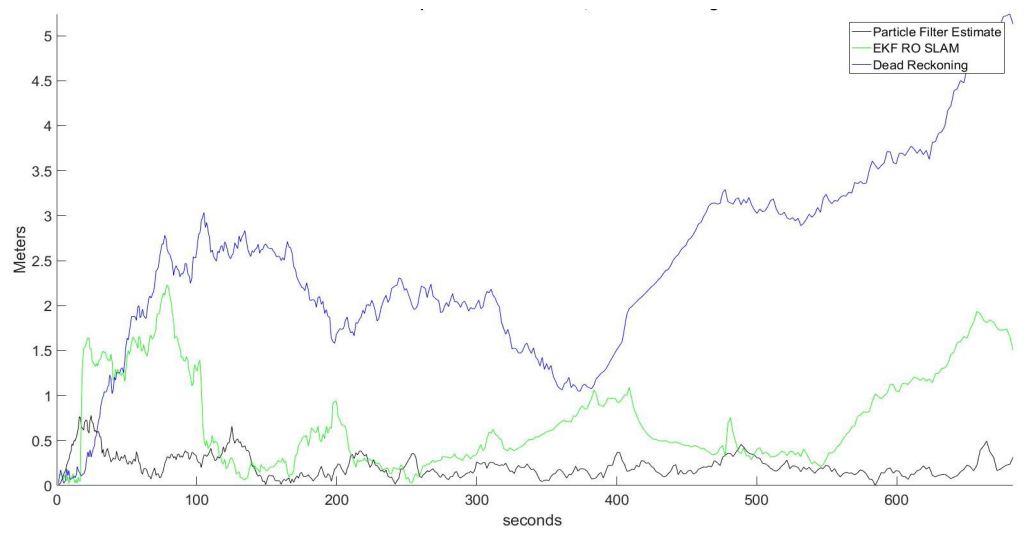

Figure 19: Error in the robot's position accumulated in a Lab environment over time. 
International Journal of Wireless \& Mobile Networks (IJWMN) Vol. 10, No. 4, August 2018

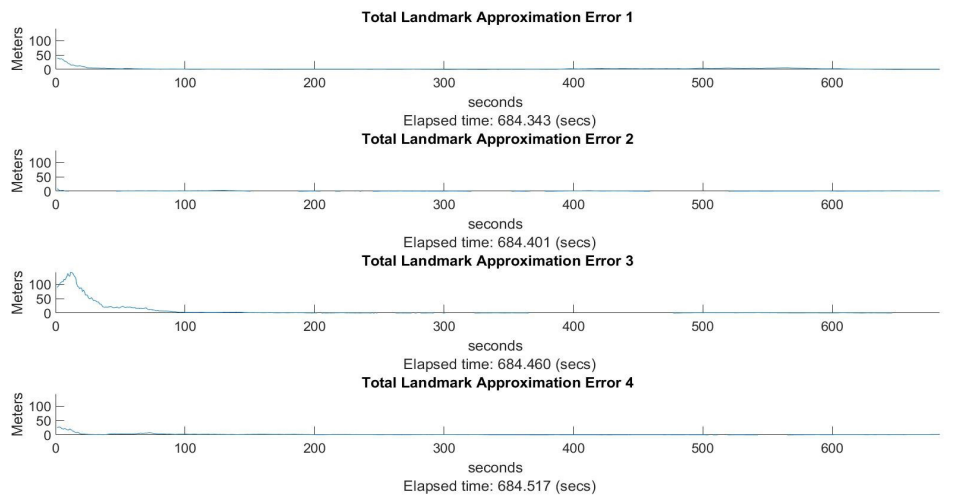

Figure 20: Error in the landmark localization in a Lab environment over time.

The furnished laboratory environment proves the most difficult of all due to the many reflective, metal desks, drawers, and chairs scattered about the room. The distribution in Figure 17 is multimodal, causing RO-EKF to perform poorly despite correct landmark initialization. The particle filter maintains similar accuracy to the other environments here as well.

\subsubsection{False Landmark Initialization}

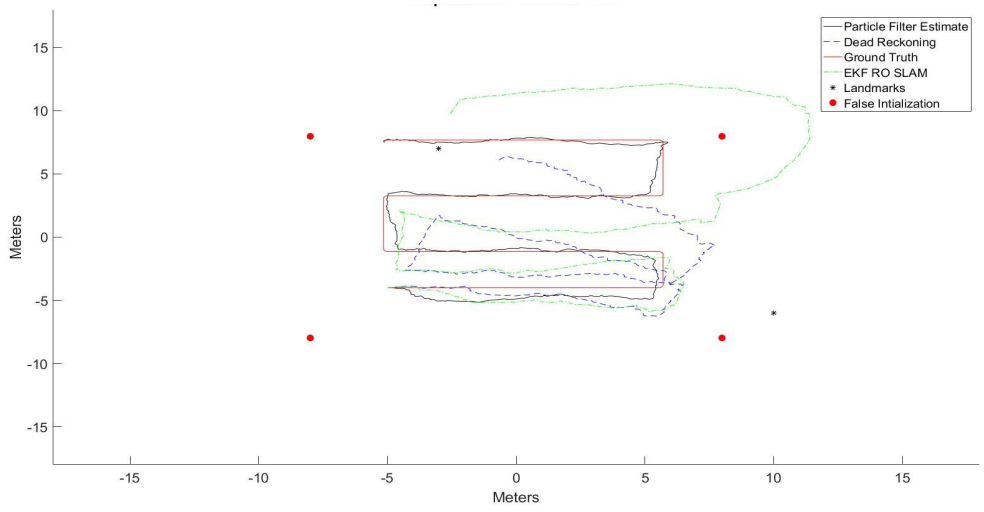

Figure 21: Error in the path followed in lab environments with false initialization.

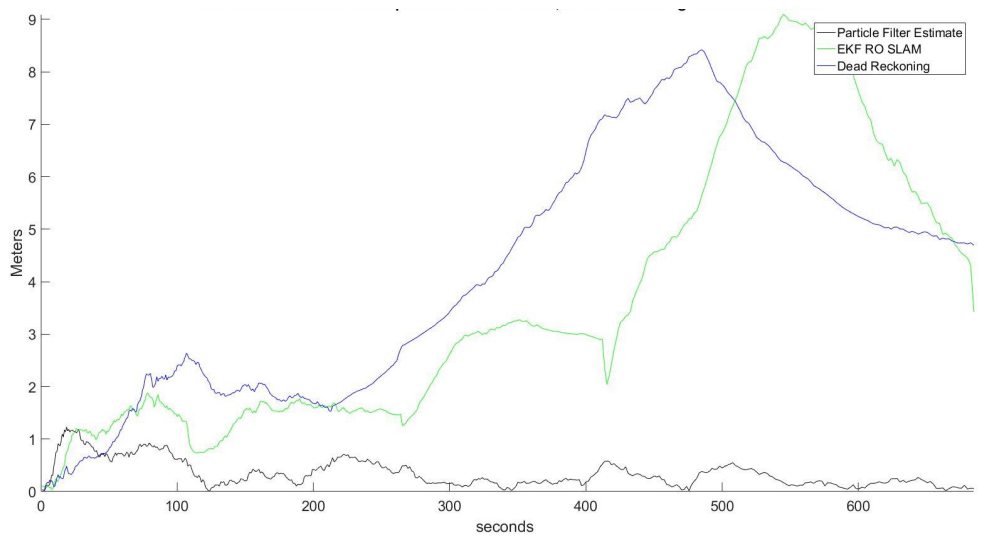

Figure 22: Error in the robot's position in a Lab environment with false initialization over time. 
International Journal of Wireless \& Mobile Networks (IJWMN) Vol. 10, No. 4, August 2018

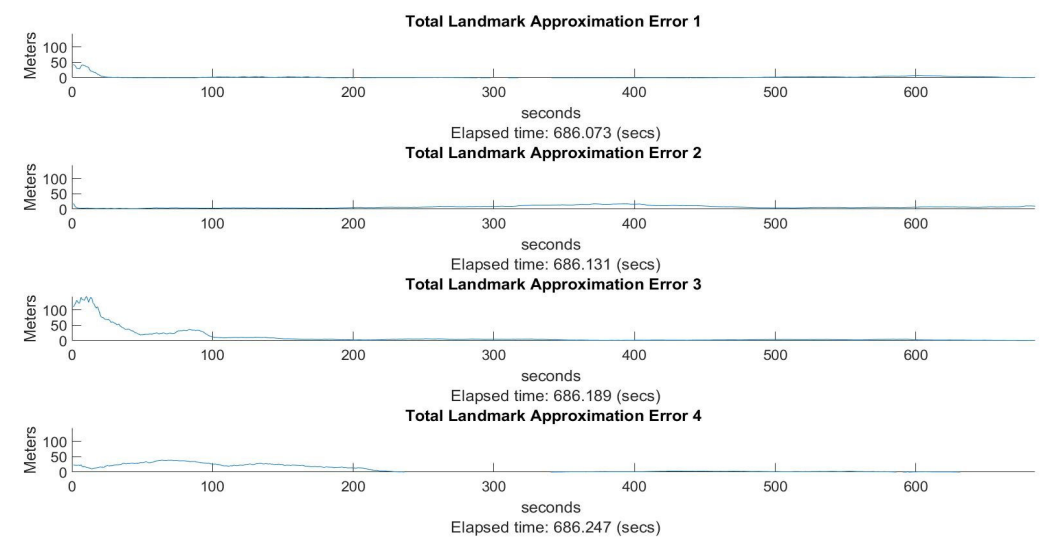

Figure 23: Error in the landmark localization in a lab environment over time.

Finally, the laboratory environment with incorrect landmark initialization creates the most nonideal RO-EKF environment, causing EKF to perform similarly to the odometry information. This is expected as the variance is so high compared to the variance of the odometry, the corrections made from the RSSI measurements are minimal. Once again, the particle filter performs as well as it does in all other conditions, along with correcting the landmark positions.

\subsection{Comparison of Environments}

As shown in Table 4.1, the EKF becomes almost comparable to the particle filter when the landmarks are correctly initialized depending on the amount of multipath it comes across as it traverses through the environment. But when falsely initialized it becomes comparable to dead reckoning. Table 4.1 contains the total error for each of these experiments for easy comparison.

Table 4.1: Comparison of accumulated robot location error over the entire path using particle filter, dead reckoning and EKF methods in different environments.

\begin{tabular}{|l|l|l|l|}
\hline RO SLAM & $\begin{array}{l}\text { Particle Filter } \\
\text { (meters) }\end{array}$ & $\begin{array}{l}\text { Dead Reckoning } \\
\text { (meters) }\end{array}$ & $\begin{array}{l}\text { EKF } \\
\text { (meters) }\end{array}$ \\
\hline Lab with true initialization & 317.566 & 2666.636 & 736.927 \\
\hline Lab with false initialization & 264.273 & 3042.626 & 1466.225 \\
\hline Large open room with true initialization & 428.855 & 701.074 & 379.047 \\
\hline Large open room with false initialization & 283.700 & 668.281 & 1363.672 \\
\hline Hallways with true initialization & 349.312 & 739.782 & 482.462 \\
\hline Hallways with false initialization & 373.271 & 1597.598 & 1552.066 \\
\hline
\end{tabular}

\subsection{Comparison regarding the number of particles used.}

Below we have presented the differences in the efficiency in using different numbers of particles. We ran the proposed particle filter method, dead reckoning, and Range Only EKF for a lab environment. We repeated the process for the particle filter using 2000, 1000, 500, 300 and 150 particles. The cases were run using the same dataset generated by the RSSI signal simulator. 


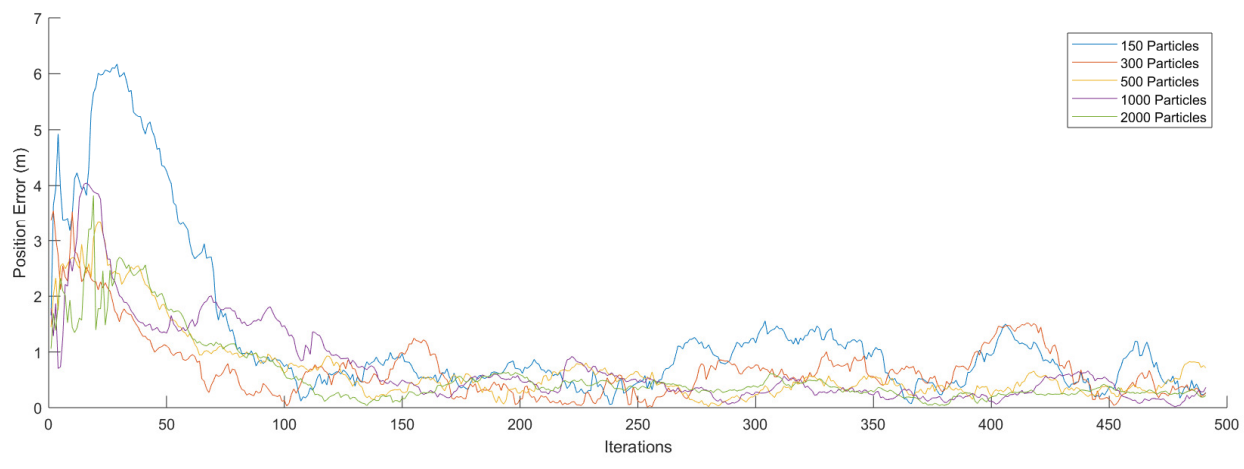

Figure 24: Particle filter convergence time for various number of particles.

As seen in Figure 24, as we reduce the number of particles we observe a trade-off in the convergence time and error incurred over the path with the particle filter with 150 particles taking the longest time to converge along with the most accumulated error incurred.

\section{Conclusions}

Here methods for RO SLAM using RSSI ranging have been explored with a focus on operation within indoor environments. These methods have a high probability of failure in highly noisy environments due to the effect multipath propagation has on range estimation from RSSI data. This causes traditional EKF RO SLAM algorithms to diverge and hence resulting in false results because multipath creates highly non-zero, non-Gaussian signal noise which violates the requirements for proper EKF functionality. Here, a particle filter based RO SLAM approach is presented to account for this issue, as particle filter approaches are appropriate for scenarios in which the noise distribution is not easily modelled.

A novel configuration for the particle filter is proposed and implemented in a simulation using realistic RSSI measurements. It is compared to RO EKF SLAM and dead reckoning in different environments varying in the degrees of multipath propagation. It is tested for both true and false landmark initializations. Performance metrics such as total error incurred over the path and landmark localization error is collected and compared. The noise from the beacons is also compared to show the distribution in the error.

In conclusion, with true landmark initialization, the EKF and the particle filter incurs comparable amounts of error depending on the multipath signals received. However, the EKF copes poorly with the non-Gaussian noise presented by multipath. When it is falsely initialized in RO EKF it barely updates the robot's position from the measurement and hence it accumulates the error incurred from odometry and becomes comparable to dead reckoning. The non-Gaussian distribution of the measurement noise further causes the landmark to be inaccurately corrected. The results also proposed to have the minimum number of particles required to represent the error distribution in the RSSI signals so that positions related to multipath propagation have some probability of being re-sampled along with the line of sight signals. In this case, anything greater than 1000 particles has negligible improvement in results.

Further research includes the implementation and evaluation of the proposed particle filter SLAM method on a physical mobile robot platform and using real- time sensor measurements. While the effect of the number of particles needed for proper distribution representation is explored here, this may not hold true for other indoor environments not evaluated in this work and the error induced by non-simulated odemetric error. Evaluation of this method in other mediums is of interest as well, apart from radio signal propagation. RO-SLAM is required for underwater 
International Journal of Wireless \& Mobile Networks (IJWMN) Vol. 10, No. 4, August 2018

autonomous vehicle applications where ultrasonic beacons must be used for landmarks in areas where the sea floor does not provide distinguishable landmarks. The multipath effect is still present in ultrasonic beacons and odometry error may even be more non-Gaussian in the face of oceanic currents.

\section{REFERENCES}

[1] M. L. Rodrigues, L. F. M. Vieira and M. F. M. Campos, "Mobile Robot Localization in Indoor Environments Using Multiple Wireless Technologies," 2012 Brazilian Robotics Symposium and Latin American Robotics Symposium, Fortaleza, 2012, pp. 79-84.

[2] S. L. Shue, "Utilization of wireless signal strength for mobile robot localization in indoor environments" (Doctoral dissertation). The University of North Carolina at Charlotte, ProQuest Dissertations Publishing, 2017.

[3] Z. Xu, K. Sandrasegaran, X. Kong, X. Zhu, J. Zhao, B. Hu, C.-C. Lin, "Pedestrain monitoring system using Wi-Fi technology and RSSI based localization", International Journal of Wireless \& Mobile Networks (IJWMN), vol. 5, no. 4, Aug. 2013.

[4] S. Sain, Modelling and Characterization of Wireless Channels in Harsh Environments, Thesis, Mälardalen University, no. 1154, pp. 136, 2011.

[5] “Two-ray ground-reflection model - Wikipedia. https://en.wikipedia.org/wiki/Two-ray_groundreflection_model. (Accessed on 03/28/2017).

[6] J. S. Seybold, Introduction to RF Propagation. John Wiley and Sons, 2005.

[7] J. Xu, W. Liu, F. Lang, Y. Zhang, C. Wang, "Distance Measurement Model Based on RSSI in WSN," Wireless Sensor Network, Vol. 2 No. 8, 2010, pp. 606-611

[8] S. Shue, L. E. Johnson and J. M. Conrad, "Utilization of XBee ZigBee modules and MATLAB for RSSI localization applications," SoutheastCon 2017, Charlotte, NC, 2017, pp. 1-6.

[9] S. Elango; N. Mathivanan, and G. Pankaj. "RSSI Based Indoor Position Monitoring Using WSN in a Home Automation Application.” Microprocessors and Microsystems. 2011; 11(4): 14-19.

[10] R. Mehra; A. Singh, "Real time RSSI error reduction in distance estimation using RLS algorithm," in Advance Computing Conference (IACC), 2013 IEEE 3rd International, pp.661-665, 22-23 Feb. 2013

[11] S. Thrun, Probabilistic robotics, Communications of the ACM, vol. 45, no. 3, 2002.

[12] S. Thrun, Particle Filters in Robotics, Proceedings of Uncertainty in AI, vol. 1, pp. 511 518, 2002.

[13] G. Grisetti, C. Stachniss, and W. Burgard, Improved Techniques for Grid Mapping With RaoBlackwellized Particle Filters, IEEE Transactions on Robotics, vol. 23, pp. 34 46, Feb 2007.

[14] G. Grisetti, R. Kummerle, C. Stachniss and W. Burgard, "A Tutorial on Graph-Based SLAM," in IEEE Intelligent Transportation Systems Magazine, vol. 2, no. 4, pp. 31-43, winter 2010.

[15] S. Shue and J. M. Conrad, Procedurally generated environments for simulating RSSI- localization applications, in Proceedings of the 20th Communications \& Networking Symposium, CNS '17, (San Diego, CA, USA), pp. 7:1 7:11, Society for Computer Simulation International, 2017.

[16] Nelyadi Samyak Shetty, "Particle Filter Approach to Overcome Multipath Propagation Error in Slam Indoor Applications" (Master's thesis). The University of North Carolina at Charlotte, ProQuest Dissertations Publishing, 2018. 


\section{Authors}

SAMUEL L. SHUE (M'14 - PhD'17) received his B.S. in computer engineering in 2011, M.S. in electrical engineering in 2015, and his Ph.D. in electrical engineering in 2017 from the University of North Carolina at Charlotte. He currently works as an embedded systems engineer for NLA Diagnostics in Charlotte and is also an adjust professor at UNCC. He is also an active member of IEEE and the IEEE Robotics and Automation Society. His research interests include localization and mapping for robotics, wireless localization, signal processing, and embedded systems.

NELYADI SAMYAK SHETTY (M'18) received the B.S degree in electronics and communication engineering from the Bangalore Institute of Technology, Bangalore, India in 2015 and the M.Sc. degree in electrical engineering from the University Of North Carolina Charlotte, North Carolina in 2018, where he worked on developing robust algorithms for robot localization using radio signals in environments that exhibit high multipath noise. His research interests include embedded systems, digital signal processing and Simultaneous Localization and Mapping algorithms for robotics

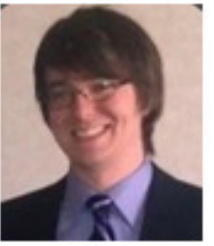
applications.

AIDAN F. BROWNE (M'95,'97 - PhD'98) received his B.S. degree in physics and mathematics from Vanderbilt University, Nashville, TN in 2008, an M.S. degree in biological engineering, an M.S. degree in electrical engineering, and a Ph.D. in biomedical engineering, from the University of Connecticut, Storrs, CT, in 1995, 1997 and 1998, respectively. He is currently an Assistant Professor in the Department of Engineering Technology and Construction Management at The University of North Carolina at Charlotte. His research interests include robotics, mechatronics, instrumentation and sensing. Dr. Browne currently serves as the Chair of the IEEE

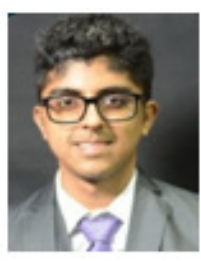
Charlotte Section. He holds memberships in the American Society of Engineering Education (ASEE) as well as the Vibration Institute.

JAMES M. CONRAD (M'87 - PhD'92) received his BS degree in computer science from the University of Illinois, Urbana, in 1984 and his M.S. and Ph.D. degrees in computer engineering from North Carolina State University in 1987 and 1992, respectively. Since 2003 he has been an Associate Professor and Professor with the Electrical and Computer Engineering Department at The University of North Carolina at Charlotte. He is the author of eight books and more than 150 articles in the areas of embedded systems, robotics, parallel processing, and engineering education. Dr. Conrad

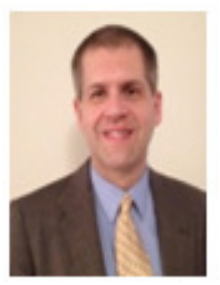
was elected to serve on the IEEE Board of Directors as Region 3 Director/Delegate for 2016-2017. He has been recognized by IEEE Region 3 and IEEE-USA with several awards for exemplary teaching and service. 